\title{
CORRESPONDENCE
}

\section{The calculation of mechanical power is not suitable for intra-patient monitoring under pressure-controlled ventilation}

\author{
Zhanqi Zhao ${ }^{1,2}$, Inez Frerichs ${ }^{3}$, Huaiwu He ${ }^{4}$, Yun Long ${ }^{4 *}$, Knut Möller ${ }^{2}$, Ary Serpa Neto $5,6,7$ \\ and Marcus J. Schultz ${ }^{5,8}$
}

(c) 2019 Springer-Verlag GmbH Germany, part of Springer Nature

\section{Original correspondence from Dr. Zhao et al. Dear Editor,}

We read with interest the paper by Serpa Neto et al. showing that mechanical power (MP) of ventilation is independently associated with in-hospital mortality and other outcomes in critically ill invasively ventilated patients [1]. Although MP might become a practical clinical outcome predictor, we would like to point out that MP calculation may not be suitable for intra-individual monitoring of ventilator-related causes of lung injury under pressure-controlled ventilation.

Serpa Neto et al. used a simplified equation for MP calculation [2], which is suitable in patients under volumecontrolled ventilation. In pressure-controlled mode, $R_{\text {aw }}$ cannot be substituted by $\left(P_{\text {peak }}-P_{\text {plat }}\right) /$ Flow and Flow by $V_{\mathrm{T}} / T_{\text {insp }}$. Further, an increase in $V_{\mathrm{T}}$ is always negatively weighted in both original and simplified calculations, while the effect of recruitment is neglected. Considering the original calculation in [2]:

$$
\begin{aligned}
& \mathrm{MP}=0.098 \times \mathrm{RR} \\
& \quad \times\left[V_{\mathrm{T}} \times\left(P_{\text {plat }}-\text { PEEP }\right) \times 1 / 2+V_{\mathrm{T}} \times R_{\mathrm{aw}} \times \text { Flow }+V_{\mathrm{T}} \times \mathrm{PEEP}\right]
\end{aligned}
$$

MP increases when the patient's respiratory system compliance $C_{\mathrm{rs}}$ increases, assuming the ventilator settings are unchanged. The effect of derecruitment on MP

\footnotetext{
*Correspondence: Iy_icu@aliyun.com

${ }^{4}$ Department of Critical Care, Chinese Academy of Medical Sciences, Peking Union Medical College Hospital, 1\# Shuaifuyuan, Dongcheng District, Beijing 100730, China

Full author information is available at the end of the article
}

calculation is highlighted in Fig. 1. During an example PEEP titration procedure, $C_{\mathrm{rs}}$ and $V_{\mathrm{T}}$ fell in the course of a decremental PEEP trial. Both the original and the simplified equations for MP calculation rendered the lowest values at zero end-expiratory pressure which would not have been the "ideal" PEEP.

We presume that inter-patient comparison using MP could be reasonable even under pressure-controlled ventilation since higher $V_{\mathrm{T}}$ is associated with higher mortality [3]. Therefore, it would be interesting to learn if the Serpa Neto et al's results [1] would have changed with a separate analysis of volume-controlled and pressure-controlled subgroups.

\section{Rebuttal from Drs. Serpa Neto and Schultz}

We thank Drs. Zhao and colleagues for their comment on our work. We completely agree that the simplified equation used for calculation of the mechanical power (MP) may not be correct for patients under pressure-controlled ventilation $(\mathrm{PCV})$. Although the applied ventilatory mode was not always recorded in the two databases we used in the analysis, we think that the majority of the patients were ventilated with volume-controlled ventilation $(\mathrm{VCV})$ as the data considered intensive care units from North America where VCV is almost exclusively used. Also, we tried to prevent patients on PCV being included in the analysis by ignoring all patients in whom plateau pressure was lacking or not reliably documented. However, since the mode was not explicitly recorded for all patients, it is impossible to run a sensitivity analysis according to ventilatory mode.

Regarding the relationship between positive endexpiratory pressure (PEEP) and MP, we also agree that 


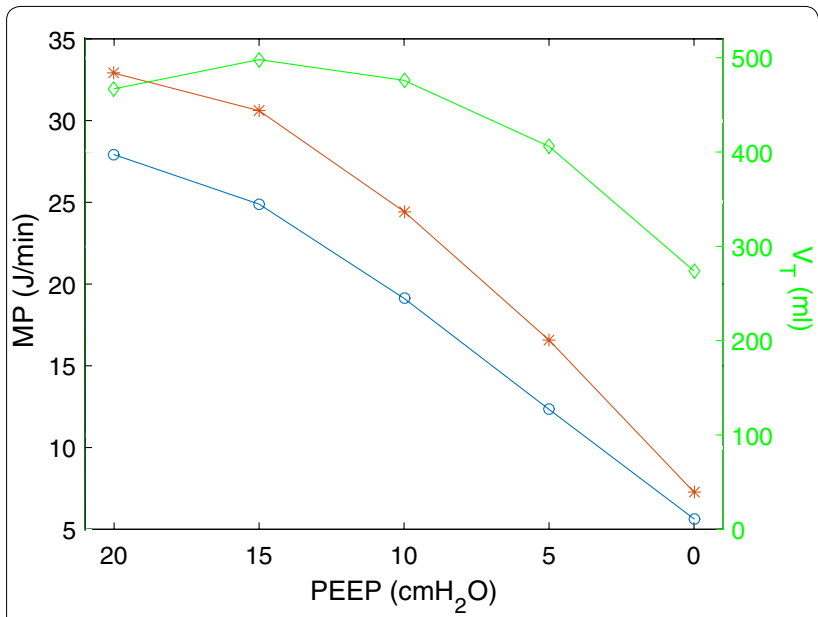

Fig. 1 An example of mechanical power (MP) calculation during decremental PEEP titration under pressure-controlled ventilation. Blue circles, MP calculated with the simplified equation; red stars, MP calculated with the original equation. (For comparison, mean tidal volumes $\left(V_{T}\right)$ at decremental PEEP steps are shown as green diamonds)

the so-called power equation may not be suitable [4]. First, there is no mechanical movement with volume displacement resulting in variation of PEEP. Second, when including PEEP into the equation, a linear and positive relationship between MP and PEEP is obtained, while a $\mathrm{U}$-shaped relationship could be more appropriate $[4,5]$. Thus, a reduction in PEEP would result in a lower MP when using the 'power equation', while lower PEEP does not necessarily reduce the risk of VILI and maybe even worsen outcomes in ARDS patients [5].

\section{Author details}

1 Department of Biomedical Engineering, Fourth Military Medical University, Xi'an, China. ${ }^{2}$ Institute of Technical Medicine, Furtwangen University, Villingen-Schwenningen, Germany. ${ }^{3}$ Department of Anesthesiology and Intensive Care Medicine, University Medical Center of Schleswig-Holstein Campus Kiel, Kiel, Germany. ${ }^{4}$ Department of Critical Care, Chinese Academy of Medical Sciences, Peking Union Medical College Hospital, 1\# Shuaifuyuan, Dongcheng District, Beijing 100730, China. ${ }^{5}$ Department of Intensive Care and Laboratory of Experimental Intensive Care and Anesthesiology (LEICA), Academic Medical Center, Amsterdam, The Netherlands. ${ }^{6}$ Department of Critical Care Medicine, Hospital Israelita Albert Einstein, São Paulo, Brazil.

${ }^{7}$ Laboratory for Critical Care Research, Hospital Israelita Albert Einstein, São Paulo, Brazil. ${ }^{8}$ Faculty of Tropical Medicine, Mahidol Oxford Tropical Medicine Research Unit (MORU), Mahidol University, Bangkok, Thailand.

\section{Compliance with ethical standards}

\section{Conflicts of interest}

The authors declared that they have no conflict of interest.

\section{Publisher's Note}

Springer Nature remains neutral with regard to jurisdictional claims in published maps and institutional affiliations.

Accepted: 14 January 2019

Published online: 6 February 2019

\section{References}

1. Serpa Neto A, Deliberato RO, Johnson AEW, Bos LD, Amorim P, Pereira SM, Cazati DC, Cordioli RL, Correa TD, Pollard TJ, Schettino GPP, Timenetsky KT, Celi LA, Pelosi P, Abreu GDM, Schultz MJ, PROVE Network Investigators (2018) Mechanical power of ventilation is associated with mortality in critically ill patients: an analysis of patients in two observational cohorts. Intensive Care Med 44:1914

2. Gattinoni L, Tonetti T, Cressoni M, Cadringher P, Herrmann P, Moerer O, Protti A, Gotti M, Chiurazzi C, Carlesso E, Chiumello D, Quintel M (2016) Ventilator-related causes of lung injury: the mechanical power. Intensive Care Med 42:1567-1575

3. Network Acute Respiratory Distress Syndrome, Brower RG, Matthay MA, Morris A, Schoenfeld D, Thompson BT, Wheeler A (2000) Ventilation with lower tidal volumes as compared with traditional tidal volumes for acute lung injury and the acute respiratory distress syndrome. N Engl J Med 342:1301-1308

4. Huhle R, Serpa Neto A, Schultz MJ, Gama de Abreu M (2018) Is mechanical power the final word on ventilator-induced lung injury? — no. Ann Transl Med 6:394-397

5. Hamlington KL, Bates JHT, Roy GS, Julianelle AJ, Charlebois C, Suki B, Smith BJ (2018) Alveolar leak develops by a rich-get-richer process in ventilator-induced lung injury. PLoS One 13:e0193934 